\title{
Next in line in next-generation sequencing: are we there yet?
}

\section{"...next-generation sequencing has its own challenges ... creating a plethora of analytical and biological considerations before these methods can be standardized."}

Keywords: drug discovery • next-generation sequencing • single-cell sequencing - technology development

Next-generation sequencing (NGS) technologies have provided us with an opportunity to explore genomes with unprecedented detail. Alongside these advancements, NGS has its own challenges (much like all technology) creating a plethora of analytical and biological considerations before these methods can be standardized. Meanwhile, encouraging single-cell sequencing has become a recent trend among scientists who are optimistic that this approach will better characterize cancer (and other similarly complex diseases) $[1,2]$. The technology necessary to sequence a complete genome from a 'single' cell has been available, but now, combined with growing popularity and mainstream acceptance, these methods are soon to become pedestrian tools, among a list of many existing NGS technologies. Will this technique be the missing link to scaffold data between genome sequencing methods, which currently contribute volumes of data but with nominal insight into therapies? To answer these questions we have chosen to review current trends in sequencing technology and relative to novel therapies.

\section{Technical barriers}

Before we invest our confidence in the concept that the genome of a single cell is informative and descriptive enough to define multifactorial diseases (i.e., cancer), the technology and science from it must be rigorously exercised and validated, a process that requires time and activity. Fundamental to all current sequencing technologies is DNA amplification and for single cell sequencing this step is critical. However, DNA amplification is well known for introducing errors and these technical imperfections are impossible to avoid [3]. High fidelity polymerase has a low error rate of $\sim 1 \times 10^{-5}$ errors per nucleotide [4]. However, this error rate is astounding when written in more simple terms as $\sim 30,000$ base substitution errors introduced per genome amplified with a single PCR cycle; multiply these errors by the number of cycles and more errors are introduced in regions with high slippage, including repeat regions, further expanding the error-rate. To overcome this hurdle one could sequence each strand multiple times, the resulting caveat: there would need to be multiple strands and even then we would introduce new errors through this amplification process and therefore expand upon natural irregularities with those presented artificially, all prior to sequencing. Additionally, single cell sequencing is complicated by the lack of experimental replicates, therefore variability between cells may be difficult to substantiate. Because of this characteristic, during the analysis of these data, variations might subse-

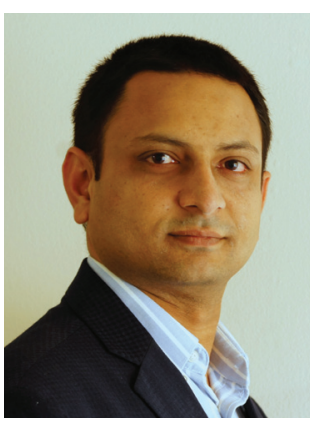

Jasmin H Bavarva

Author for correspondence: Biospecimen Research Group, Leidos Biomedical Research, Inc. (Formerly SAIC-Frederick), Frederick National Laboratory for Cancer Research (NIH/NCI), Rockville, MD 20852, USA jasmin.spu@gmail.com

Megha J Bavarva

Enusha Karunasena

Virginia Bioinformatics Institute, Virginia Tech, 1015 Life Sciences Circle, Blacksburg, VA 24061, USA 
quently pass as a 'natural' characteristic of the cell with no clear scientific procedure to determine the validity of this variation without reasonable controls (i.e., biological replicates). The next resulting challenge would be a question of how representative our determined sequence data is to the real sequence? Unfortunately, this problem persists in existing NGS technologies but also with Sanger sequencing, which is more than two decades old and considered a gold standard $[5,6]$. Accuracy rates of $100 \%$ are impractical to expect, thus biological interpretations are dependent upon statistical methods to compensate for errors; which in actuality will solve problems in interpretation but will not address the sequencing errors produced. Additionally, NGS technologies and their data analysis methods are still at a nascent stage, therefore, until these systems are matured and standardized, biological interpretations should be evaluated with consideration [7].

\section{Biological complexity}

Underlying, these technical complications are biological challenges resulting from natural genetic variants introduced into the human gene pool through increasing rates of population growth, decreasing global rates of morbidity and mortality, longer life-spans, deferred childbearing and environmental factors (i.e., pollutants, climate-change, biological and chemical agents). The growth of rare variants is predicted to be $5-14 \%$ per generation and is accelerating [8]. In fact, as sample size increases the number of observed variants grows. Hypothetically, this suggests that genetic variations and genetic maps established today may be dated material tomorrow. Yet, one could also argue that these genetic data are informative and do provide insight into disease-linked mutations. However, common genetic variants are known to have only modest effects, but if mutations are introduced in each protein-coding gene at a rate of $\sim 1 \times 10^{-5}$ per generation for nonsynonymous variants, we would then expect every gene to harbor functionally important variants [9]. A recent study reports that 35 unique single-nucleotide variations (SNVs) are newly acquired in coding sequences after 20 cell divisions by a single cell, which suggests a rate of 1.75 new SNVs in the coding sequence per cell, per cell division [3]. Therefore, genome-wide, there could be as many as 116 new SNVs/cell/cell division. Furthermore, identifying the genetic basis of complex traits through exome sequencing has not produced results with a level of confidence to suggest causation or function, and it has been suggested that on the order of over 10,000 samples may be required to reach sufficient statistical power [9]. Also, exome represents only $\sim 1.5 \%$ of the total genome and characterizing a disease based on $1.5 \%$ of the genome (on the basis of what is currently defined as the functional regions) seems debatable.

Our recent work describes the dynamic nature of the human exome and identified a wide range of intraindividual variation rates $\left(9.6 \times 10^{-7}\right.$ to $8.4 \times 10^{-6} \mathrm{bp}^{-1 \text { year-1 }}$ for SNVs) [10]. This report further described the discrepancies in current sequencing and analytical approaches that rely on reference human genomes for interpretation and variant calling, and consequences for not accounting for the changing aspects of an individual genome and environmental influences on individual bases.

66 "...we are now accelerating towards sequencing human genomes on a single cell basis, creating billions of genome sequences ... in the hope that this will better characterize diseases and result in therapies. However, we may be in a race to amplify error instead of information."

Certainly, one could correctly argue that the coding region of the genome is a foundation to build upon, given reproducible mechanistic and hypothesis-driven insight that have been vetted exhaustively through biological analysis. It is perhaps the organic process of genome analysis and subsequent insight - prior to the accelerated pace of NGS advancements - that may, again, provide the corrective measures for current technologies, which are changing faster than the biological insight obtainable through them. With such considerations that remain to be addressed we are now accelerating towards sequencing human genomes on a single cell basis, creating billions of genome sequences (i.e., thousands of cells per tumor mass from an individual multiplied by thousands of individuals per specific cancer population) in the hope that this will better characterize diseases and result in therapies. However, we may be in a race to amplify error instead of information. There will be exponential growth of natural and technologyinduced genetic variations that we are sure to base our scientific interpretations on while the impact of genetic selection pressure and environmental influences may be inopportunely vacant from these analyses. As scientists who rely upon these technologies and interpret genomic variation that may provide insight into cancer and other diseases, we ask these questions of ourselves to account for our own scientific endeavors and conclusions. Thus, the goal is not to create alarm or pessimism (or that the 'pot is calling the kettle black'), but rather introspection and reflection on an arena of methods that are here to stay and therefore require thoughtful and responsible evaluation, given the phenomenal pace of technological change (otherwise unprecedented in the biological sciences); and more importantly the 
scientific and medical consequences interpreted from these data.

There are monumental questions to consider: such as, are we prepared to have billions of obsolete genome sequences before we have a full appreciation for what is currently available? Are we confident in the goal we are racing towards? A recent report described identical DNA sequences, which resulted in contrasting cell fate whether the cell was cancerous or normal; such studies warrant our consideration for what other biological contributions affect cells [11]. The US FDA's Center for Biologics Evaluation and Research (CBER) shared that the first action approval rate of new molecular/biologics license applications (NMEs/BLAs) are at an all-time high (74\% in 2011 and $72 \%$ in 2012) and median total time to approval is at an all-time low (5.8 months for priority and 10 months for standard NMEs/BLAs). However, despite all these measures to foster new drug development, new molecular entity approval remains unaffected suggesting issues of efficacy [12]. Better success rates for new drug development were achieved in the previous decade - prior to the advent of technology-driven research (Figure 1). Generally, there is a weak relationship with technologi- cal advancements in the previous decade and actual drug development. Drug development is stable if not declining $\left(y=-0.5391 x+35.711, R^{2}=0.115\right)$, while the use of technology is exponentially growing ( $y=241.48 \mathrm{x}+$ $\left.1861.4, R^{2}=0.965\right)$ suggesting that they may not have sufficient correlation (Figure 1). Therefore, now may be the opportunity to re-focus and provide balance between basic researches with technology driven science, while we assess the value of 'big-data'.

At this precipice there is also scientific opportunity. We cannot afford to ignore anomalies introduced through technology if the end goal is to introduce better healthcare and science. We cannot minimize interpretations introduced to fundamental science if they are based on error-prone technologies. One might wonder where the proposed solutions to these problems are and appropriately suggest that some of these issues are complications naturally encountered with the growing pains of scientific venture and progress. To provide answers to these questions, we are proposing the following fundamental concepts: first let's delimit a disease that we have the greatest opportunity to gain poignant insight for via genome sequencing on a per

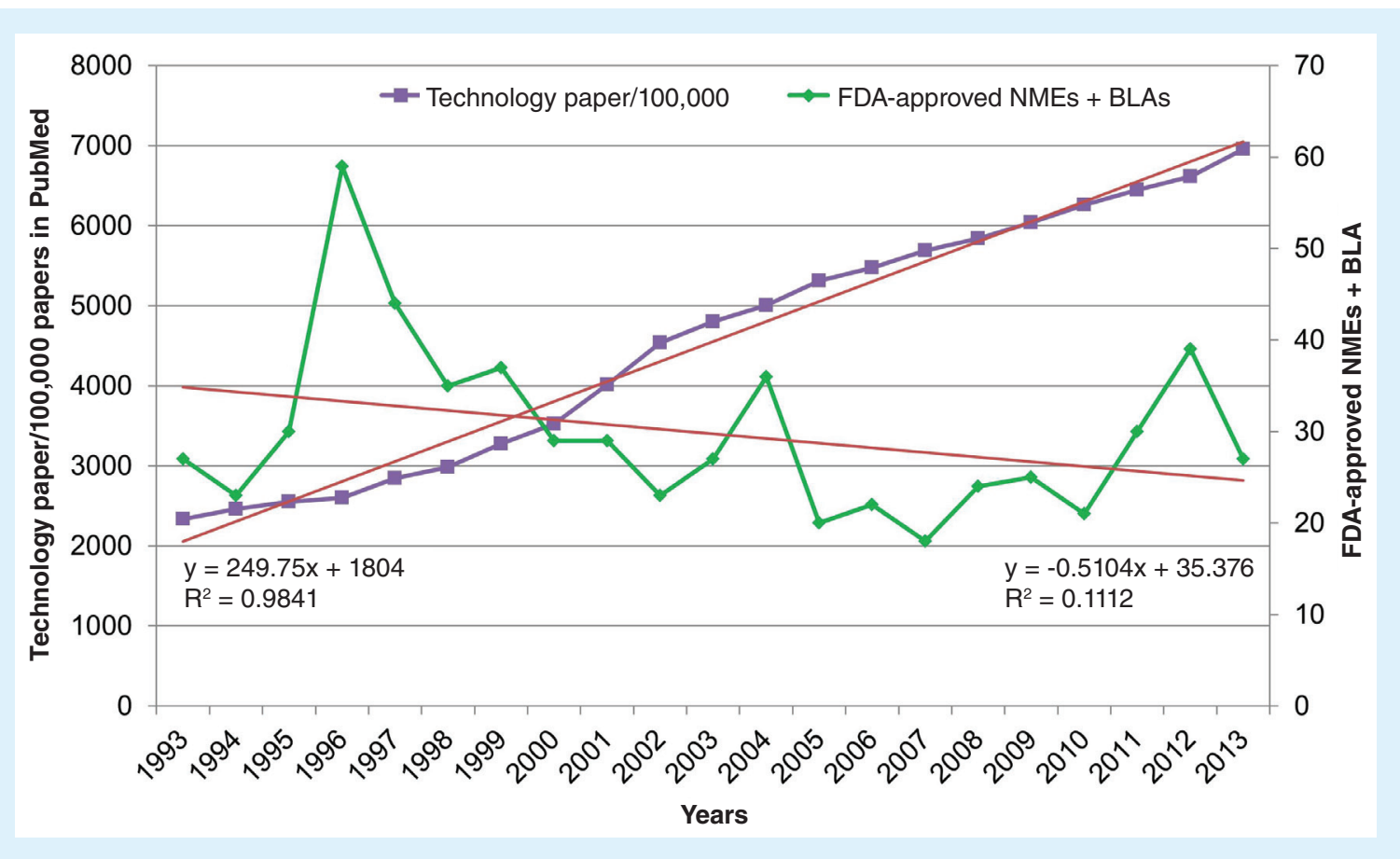

Figure 1. Gross correlation of number of published studies with term 'technology' and number of approved new molecular entities + biologics license applications per year. Line graph indicating the correlation of published manuscripts with technology versus total NMEs, plus new BLAs approved by the US FDA per year (these data are described by a green line). Counting papers with the term 'technology' per 100,000 papers in PubMed is a gross bibliometric method of overall trend (purple colored line), extracted using a web-based tool developed by Alexandru Dan Corlan (available at www.webcitation.org/65RkD48SV). Red lines are linear trend lines accompanied by $\mathrm{R}^{2}$ and slope values.

BLA: Biologics license application; NMEs: New molecular entity.

Please see color figure at www.futuremedicine.com/doi/pdf/10.2217/pgs.14.161 
cell basis, while developing methods to circumvent error and focusing on to better understand $\sim 98 \%$ of the poorly understood genome. We can then begin to appreciate the complexity of a disease relative to the available technology, which will lend itself to appropriate hypothesis generation. A development of technologies that have practical applications, advance scientific understanding, and ultimately improve healthcare quality is essential to our success. But, technology exists to support scientific discovery not vice versa.
Financial \& competing interests disclosure

The authors have no relevant affiliations or financial involvement with any organization or entity with a financial interest in or financial conflict with the subject matter or materials discussed in the manuscript. This includes employment, consultancies, honoraria, stock ownership or options, expert testimony, grants or patents received or pending, or royalties.

No writing assistance was utilized in the production of this manuscript.

\section{Executive summary}

- The use of technology is increasing in research but its contribution to our understanding of basic biological processes is yet finite.

- The race for single cell sequencing may provide marginal insight into multifactorial diseases (such as cancer) unless appropriate standardization measures for the resulting sequencing data are developed, the inherent error, and better accounting and understanding of biological complexity is in place.

- Technology development has weak correlation with the drug development.

- Next-generation sequencing technologies have accelerated the growth of biological data and will continue to contribute to these advances; however, the evaluation of these rapidly modifying technologies relative to their scientific contributions are necessary, given our growing reliance.

\section{References}

1 Landau DA, Carter SL, Stojanov P et al. Evolution and impact of subclonal mutations in chronic lymphocytic leukemia. Cell 152(4), 714-726 (2013).

2 Navin N, Kendall J, Troge J et al. Tumour evolution inferred by single-cell sequencing. Nature 472(7341), 90-94 (2011).

3 Zong C, Lu S, Chapman AR, Xie XS. Genome-wide detection of single-nucleotide and copy-number variations of a single human cell. Science 338(6114), 1622-1626 (2012).

4 Eckert KA, Kunkel TA. High fidelity DNA synthesis by the Thermus aquaticus DNA polymerase. Nucleic Acids Res. 18(13), 3739-3744 (1990).

5 Chen CY. DNA polymerases drive DNA sequencingby-synthesis technologies: both past and present. Front. Microbiol. 5, 305 (2014).

6 De Leeneer K, De Schrijver J, Clement L et al. Practical tools to implement massive parallel pyrosequencing of PCR products in next generation molecular diagnostics. PLoS ONE 6(9), 30 (2011).
7 Bavarva JH, McMahon W, Bavarva MJ, Karunasena E, Garner HR. Standardizing next-generation sequencing experiments and analysis methods. Clin. Chem. 58(12), 1720-1722 (2012).

8 Keinan A, Clark AG. Recent explosive human population growth has resulted in an excess of rare genetic variants. Science 336(6082), 740-743 (2012).

9 Kiezun A, Garimella K, Do R et al. Exome sequencing and the genetic basis of complex traits. Nat. Genet. 44(6), 623-630 (2012).

10 Bavarva JH, Tae H, McIver L, Karunasena E, Garner HR. The Dynamic Exome: acquired variants as individuals age. Aging 6(6), 511-521 (2014)

11 Kreso A, O’Brien CA, van Galen P et al. Variable clonal repopulation dynamics influence chemotherapy response in colorectal cancer. Science 339 (6119), 543-548 (2013).

12 Mullard A. 2012 FDA drug approvals. Nat. Rev. Drug Discov. 12(2), 87-90 (2013). 